\title{
An autonomous fallers monitoring kit: release $0.0^{\star}$
}

\author{
Enrique de la $\mathrm{Cal}^{1}$, Alvaro DaSilva ${ }^{2}$, Mirko Fáñez ${ }^{2}$, Jose Ramón Villar ${ }^{1}$, Javier \\ Sedano $^{2}$, and Victor Suárez ${ }^{3}$ \\ 1 University of Oviedo, Computer Science Department, \\ Oviedo, Spain \\ \{delacal, villarjose\}@uniovi.es \\ 2 Instituto Tecnológico de Castilla y León, Pol. Ind. Villalonquejar, \\ 09001, Burgos, Spain \\ mirko@mirkoo.es, javier.sedano@itcl.es, ada@ubu.es \\ 3 University of Oviedo, Control and Automatica Department, \\ EPI, Gijón, Spain \\ vmsuarez@uniovi.es
}

\begin{abstract}
Fall is one of the main causes of the reduction of the Quality of Live in Elderly, since it has a high impact in mortality rate as well as in the probability of different disabilities like Fractures, head injuries, etc, and mainly a loss of confidence. Thus, a monitoring instrument to follow elderly activity is required and the most common non-invasive and easy-to-use are based on wearable devices. This work presents our first prototype of an autonomous, low-cost and easy-to-use elderly activity monitoring kit suitable to be deployed in nursery houses. The prototype is composed of: i) our own data analytics web application deployed in a low cost server located in our laboratory (SERVER) and ii) a transparent plastic case with a set of 10 commercial smartbands (WD), their respective charging docks, one NUC (Next Unit of Computing) as data gateway, one Wi-Fi router and one LTE router. Concerning the case, we can state that each WD has built-in a 3DACC (3DA), a Gyroscope (GY) and a wrist Heart Rate sensor (HR). Besides each WD is running our own software instead the pre-built commercial one in order to achieve two issues: recording high frequency data $(1 \mathrm{~Hz}$ for $\mathrm{HR}$ and $10 \mathrm{~Hz}$ for $3 \mathrm{DA}$ and GY) and optimize the battery life (18 hours with $10 \mathrm{~Hz}$ continuous sensors recording). Finally, a first trial of the presented kit is being carried out in a Nursery House in Burgos (Spain), and the participants enrolled criteria have been designed by a gerontologist from the Diputación de Burgos (Spain).
\end{abstract}

Keywords: Falls in Elderly, Wearable sensors, Fall Detection

\section{Introduction and motivation}

Healthy aging is one of the main challenges that is asking industry and researchers for products and services to assist people remain independent, produc-

\footnotetext{
* Supported by University of Oviedo and ITCL
} 
tive, active and socially connected for longer. Fall is one of the main causes of the reduction of the Quality of Live in Elderly, since it has a high impact in mortality rate as well as in the probability of different disabilities like Fractures, head injuries, etc, and mainly a loss of confidence. Besides, the influence in the Healthcare public and private systems is high, considering both, direct and indirect costs. Thus, non-invasive tools to monitor and analyse Elderly activity are required. The most common non-invasive and easy-to-use tools to measure the activity in elderly are the wearable devices. This work presents the first prototype of an autonomous, low-cost and easy-to-use elderly activity monitoring kit suitable to be deployed in nursery houses. Even when the data analytics web app is not part of this contribution, we can state that we have the preliminary releases of a suite of fall detection algorithms based on different machine-learning paradigms $[14,16]$. Finally, a first trial of the presented kit is being carried out in a Nursery House in Burgos (Spain), and the participants enrolled criteria have been designed by a gerontologist from the Diputación de Burgos (Spain). This work is structured as follows. Next section includes the state of art about fall detection platforms, while the design issues of the falls monitoring kit is explained in 3. Experimentation and the discussion on the results are coped in section 4 . Finally, conclusions and future work is included in section 5 .

\section{State of art}

In our previous work [22], a specific platform was presented for the monitoring and detection of epilepsy, and it analyzed the platforms for this purpose in the literature [11], both those that use WD and those that use sensor networks corporal [13]. Traditionally WDs are used to collect data corresponding to biomedical variables or to obtain feedback from patients, either by doing calculations in the WD [19] or through a cloud service [18]. There exist several works in the literature where WD are used to monitorize falls [12]. Also in very a preliminary release of our proposal we included a comparison between our wearable-devices and the Apple Watch (Apple3), where it can be stated that battery last of our devices is more than the doble of the Apple Watch [9].

Sensors for Fall Detection The solutions for the detection of falls can combine different types of sensors such as a barometer and an inertial [20], 3DACC and gyroscope [21], 3DACC and smart tiles [8] or 3DACC and barometer in the neck [3]. However, 3DACC is the most widespread solution in the literature [10]. Different solutions have been proposed for the detection of falls [4], but most of them have the common characteristic that the device has been placed on the hip or on the chest, since it is easier to detect at these locations [7].

The hip location is valid for patients with severe disability, but forcing the use of a belt in conjunction with dresses (in the case of women), may not be valid for healthy patients. 
Public datasets with genuine One of the main challenges in the field of the detection of human activity (HAR - Human Activity Recognition), consists of the provision of contrasted datasets for the validation of the new detection algorithms. Among the best-known public datasets we can point out the following: UMA Fall [5] (simulated falls with a sensor located on the wrist), UNIOVIEpilepsy [23] (epileptic seizures and other activities with a sensor located on the wrist), DaLiac [17] (multiple activities from running to riding a bike, with sensors located in different places) and FARSEEING dataset [2] (simulated falls with sensor located on the thigh).

The present work aims to create a public dataset with genuine fall data from older people.

\section{The proposal}

This proposal consists of three main elements: 1) A box-kit containing all the WD and other devices necessary for the storage of the collected data; 2) the WD App responsible of recording sensors data with the desired frequency; and 3) a Web Application for data exploitation.

\subsection{The box}

The box-kit consists of the following elements: a) 10 WD for participants biometric data collection. The chosen WD is the Samsung Gear Fit 2 band, with a wearable app developed for Tizen OS, b) 1 Intel NUC for data storage. Model BOXNUC6CAYH, this mini-pc is powered by an Intel Celeron J3455 CPU and 8GB of RAM. It has 480 GB of SSD storage, c) 1 TP-Link Wi-Fi Router for WD-NUC connections. Model TP-Link TL-WR840N, being able to transmit at 300 Mbps, d) 1 4G Huawei Router for outside access to the NUC data. Model Huawei B525 4G/LTE, with a 5GB per month SIM card, e) 1 Multiple-socket outlet for supplying power to all the devices. ORICO Super Charger with 6 outlets and 5 USB $5 \mathrm{~V} / 8 \mathrm{~A}$ charging ports, and f) 2 3-Ports USB Chargers for charging all the remaining WD. RAVPower Fast USB Chargers 5V/6A.

All these items are assembled on a plastic box for easy transport (10€). The cost of the entire assembly is $478 €$ (NUC, routers, chargers and box) + $25 € /$ month (Mobile data plan) $+125 € /$ participant (each WD). There are no known limitations on the number of participants this system supports (apart from the obvious: box size and USB charging ports; the more participants, the more physical space is required for each WD charging station and more USB chargers are needed). We have developed a system starting with 10 participants for one nursery house, but with plans to add more kits to the system in other nursery houses.

Each WD is able to record up to 18 hours/day of sensors-data with its 200 $\mathrm{mAh}$ battery, which is approximately $90 \mathrm{MB}$ /day for each participant. So, for 10 participants recording 18 hours each day, the NUC with its 480GB of SSD storage is able to keep approximately up to 17 months of data (with space for 
the Windows 10 NUC's OS). If for any reason the NUC isn't available, each WD is able to keep 22 days of data with its $2 \mathrm{~GB}$ of free internal storage.

Through the LTE connection, daily data summaries are sent using a scheduled task via FTP to a lab server, in which there is a web application prepared for viewing these summaries. Them contain aggregation information of the data recorded on each WD, which allows you to verify remotely that the system is working properly and the adequacy of the data recorded for each participant, without sending all this data through a mobile connection, that would require to contract an Internet mobile flat rate and more costs. The retrieval of the raw data from the NUC can be done in person when necessary (up to once every 17 months), moving all data to a removable device.

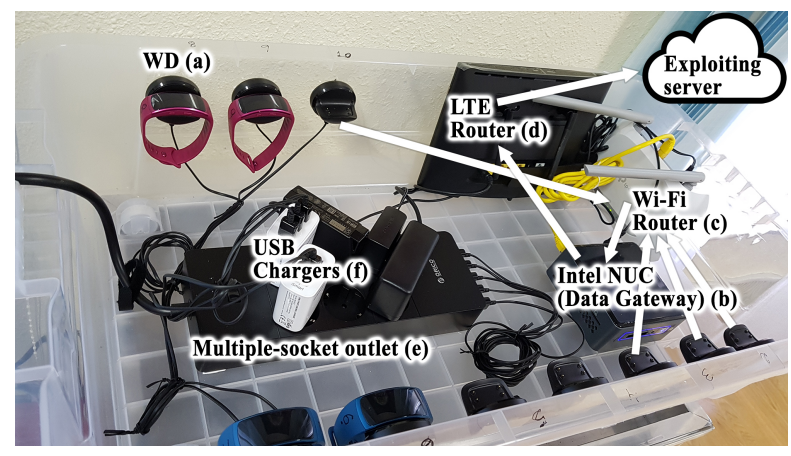

Fig. 1. The fallers monitoring kit 0.0

\subsection{The Wearable App}

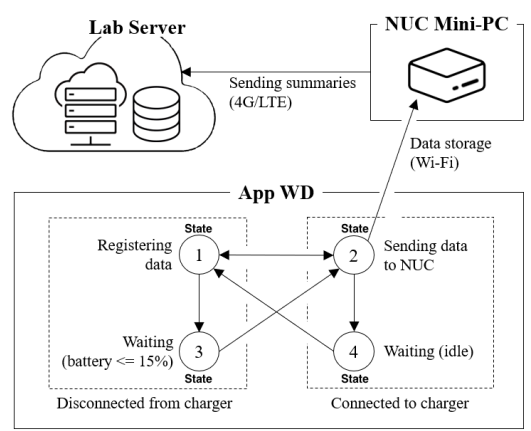

Fig. 2. States diagram for the app WD and successive data manipulation. 


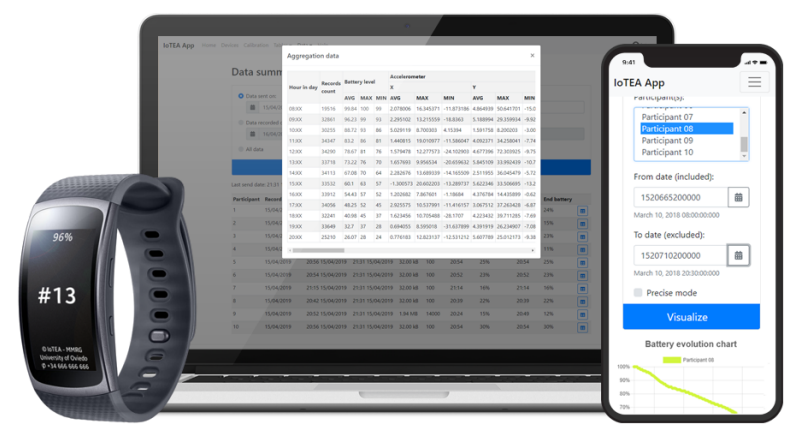

Fig. 3. WD application (left), Data Visualization Web App on both desktop and mobile (right).

The WD that has been chosen is a Samsung commercial device equipped with optical HR, 3DA and GY sensors, as well as WiFi connectivity. The WD configuration has been customized in order to minimize battery consumption, getting rid of device features that were not focused on this platform.

For this particular WD, which runs under Tizen OS version 2.3.1, a system has been developed for the continuous recording of data, which clearly improves the native solution of the manufacturer both in battery consumption and sampling frequency. This system consists of: i) an application (the Launcher) responsible for managing (launching, stopping, pausing, etc.) the data recording and transmission services. This application is password-protected, only available to administrator users. ii) A Watch-Face, responsible of showing anytime informative data to the user (participant ID, Battery \%, etc), as well as guaranteeing that the data recording and transmission services are operative (Figure 3). iii) The service responsible for collecting the data from the sensors with the frequency set $(1 \mathrm{~Hz}$ for $\mathrm{HR}$ and $10 \mathrm{~Hz}$ for the $3 \mathrm{DA}$ and GY sensors) and store them, locally, in a database. iv) The data transmission service, responsible for sending the collected data through the Wi-Fi connection via FTP to the data gateway (the NUC device).

Figure 2 shows a state diagram with operational detail of the system: state1) the sensors are recording data continuously while the device is ON and worn by a participant, state2) Likewise, it sends the collected samples to the NUC via FTP using the Wi-Fi connection when the WD is placed on the charging base, state3) Once all data from the WD has been downloaded into the data gateway, the WD App goes idle, state4) until it is removed from the charger the next morning, to be placed on the participants again (state 1). The WD App is able to do this autonomously, even to stop recording data from the sensors when reaching a battery level lower than 15\% (State 3). Thanks to this behaviour, the nurses only have to worry about picking up the WD from the chargers at the beginning of the day and placing them on the participants, and at the end of 
the day taking them off and putting them back to the chargers. This allows a fully automated operation, without no interaction with the WD.

\subsection{The Data Visualization Web App}

To exploit easily with all the collected data (each WD registers more than half a million of samples per day), a web application (see Figure 3) has been developed, which allows: i) the visualization and filtering of the summaries of the data registered in the WD that are sent each day from the NUC, ii) The generation of graphs and other summarized information from the data previously moved from the NUC to the lab server. This web application would also allows in the future to carry out a calibration process of the activity of each participants and the sensors measures. This calibration consist in a series of specific activities that would be carried out by the participants while sensors data is being registered with the WD. This calibration would help when designing and training machine learning models using participants' data. This web application is deployed on the data exploitation server, located in the remote laboratory. It is prepared to receive data from multiple nursery houses at once, simply by having the NUCs from each of the places send the summaries to the same web server. Even when the data analytics process is not part of this contribution, we can state that we have the preliminary releases of a suite of fall detection algorithms based on different machine-learning paradigms [15].

\section{Preliminary results}

Falls are defined as involuntary events that make one lose balance and find the body on the ground or other firm surface that stops it $\left(\mathrm{WHO}^{4}, 2012\right)$. Falls are an important cause of disability in the elderly and, at their Once, one of the adverse outcomes of frailty. Approximately $30 \%$ of people over 65 and $50 \%$ of those over 80 fall at least once a year[1].

\subsection{Inclusion and exclusion criteria}

Among the 150 patients living in the Nursery House "Fuentes Blancas" (Diputación de Burgos, Spain), 10 participants have been enrolled (table 1 shows the exclusion and inclusion criteria) in the first phase of the presented falls monitoring kit: 8 fallers and 2 healthy participants (participants 4 and 8 ). The fallers have an average fall rate of one event per 15 days. Table 2 shows the features and comorbilites for each participant.

Comorbidity implies an increase in risk factors involved in falls and an increase in mortality due to various factors, including polypharmacy. The Charlson Comorbidity Index [6] considers, based on the pathologies potentially responsible for death, the prediction capacity based on the score obtained plus a correction

\footnotetext{
${ }^{4}$ World Health Organization
} 
Table 1. Inclusion and exclusion criteria

\begin{tabular}{l|l}
\hline Inclusion & Exclusion \\
\hline Having suffered at least one fall in the last year. & Dementia or Cognitive Impairment. \\
\hline Stable cognitive situation with MMSE greater than 26/30. & Functional or physical instability. \\
\hline Maintenance of sensory-perceptual abilities. & Life expectancy less than six months. \\
\hline Ability to move autonomously. & \\
\hline
\end{tabular}

Table 2. Features of the enrolled participants: IdParticipant, Gender, Age and Comorbilites

\begin{tabular}{|c|c|c|c|}
\hline Id & Gender & Age & Comorbilites \\
\hline 1 & Male & 88 & Left amaurosis, diabetes, ulcus, prostatism, hypertension, renal failure, ACXFA \\
\hline 2 & Male & 78 & Diabetes, hypertension, heart disease, aplastic anemia, duodenopancreatectomy \\
\hline 3 & Male & 86 & $\begin{array}{l}\text { Diabetes, hyperuricemia, brucellosis, ulcus, osteoarthritis, spastic colon, laminectomy, } \\
\text { cholecystectomy }\end{array}$ \\
\hline 4 & Male & 76 & Hypertension, diabetes, renal failure, diabetic nephropathy \\
\hline 5 & Female & 89 & $\begin{array}{l}\text { piliartrosis, diabetes, hypertension, total knees prosthesis, hyperlipemia, hepatic hydatid } \\
\text { cyst, diabetic retinopathy, diabetic nephropathy, ulcus, hypothyroidism }\end{array}$ \\
\hline 6 & Female & 91 & $\begin{array}{l}\text { Glaucoma, AC X FA, knee prostheses, enf Crohn, shoulder subluxation, chronic renal } \\
\text { failure, varicose veins }\end{array}$ \\
\hline 7 & Male & 88 & $\begin{array}{l}\text { Prostatism, hypertension, nephrectomy, COPD, dyslipidemia, arthritis, AC x FA, knee } \\
\text { prosthesis, olecranon fracture, chronic renal failure, intermittent claudication }\end{array}$ \\
\hline 8 & Male & 83 & $\begin{array}{l}\text { Diabetes, Hypertension, Hyperlipidemia, Aortic aneurysm, COPD, Goiter, polycystosis } \\
\text { hepatica, nephrolithiasis }\end{array}$ \\
\hline 9 & Female & 85 & Hypertension, fx, humero, syncope, bilateral aphakia, delusional disorder, hearing loss \\
\hline 10 & Female & 98 & $\begin{array}{l}\text { Anemia, endometrial carcinoma, mild cognitive impairment, ACXFA, renal failure, PE, } \\
\text { bilateral DVT, Fx colles, ulcus }\end{array}$ \\
\hline
\end{tabular}

factor for the number of years. The taking of more than five medications constitutes a Geriatric Syndrome that we call Polypharmacy. The intake of several drugs increases the risk of the adverse effects of the drugs and the changes derived from normal aging those of pharmacodynamics. The Potential Toxicity Scale (PTS) quantifies with a correction factor the taking of drugs and the possible implication in falls.

Table 3 includes the computation of the typical risk indexes for elderly: CCI, Aid, Treatments, BARTHEL and MMSE. The two healthy participants (id = $4,8)$ score a BARTHEL index of 90 and 95 respectively, that a shows a very good physical state. The remaining indexes will allow to correlate the state of the participants and the physical evolution of participants (considering falls and other kind of activities).

\subsection{Numerical results}

This is only a very preliminary resume of the two first weeks of working. The monitoring kit has been running from 9am to 9pm with all the 10 participants.

The WDs were able to record 12 hours of continuous data (one day) as planned. At the end of these 12 hours, the battery life was around $25 \%$ charge (see Figure 4), that is a very good performance. In the summaries sent from the NUC to the Lab SERVER, we can also obtain average information for all the sensors, aggregated by hour (see HR sensor data in Figure 5). It can be observed that in the period between $2 \mathrm{pm}-4 \mathrm{pm}$ there is a valley in HR curve due to the nap time for the most of the participants. Two important issues were: i) that all the 
de la Cal E. et al.

Table 3. Risk factors for the enrolled participants: Id (IdParticipant), CCI (The Charlson Comorbidity Index), Aid (cane or walker), Treatments, PTS(Potencial Toxicity Scale), BARTHEL (Assessment of physical disability), MMSE (Mini-Mental State Exam)

\begin{tabular}{l|l|l|l|l|l|l}
\hline Id & ICC & Aid & Treatments & PTS & BARTHEL & MMSE \\
\hline 1 & 6 & cane & Duodart, digoxin, enalapril, sintrom, xelevia & 5 & $75 / 100$ & $27 / 30$ \\
\hline 2 & 3 & & zomarist, sevikar & 3 & $80 / 100$ & $26 / 30$ \\
\hline 3 & 3 & cane & $\begin{array}{l}\text { Omeprazole Pregabalin, xelevia, mirtazapine, xarelto, bisoprolol, } \\
\text { zuranpic, seretide, mixtard, feliben }\end{array}$ & $70 / 100$ & $28 / 30$ \\
\hline 4 & 8 & & atrovastatin, lantus, sevikar, velmetia & 4 & $90 / 100$ & $28 / 30$ \\
\hline 5 & 11 & walker & omeprazole, diliban, eutirox, inegy, vesicare, mixtard & 4 & $65 / 100$ & $26 / 30$ \\
\hline 6 & 6 & cane & Omeprazole Openvas, sintrom, travatan, tardyferon & 2 & $70 / 100$ & $26 / 30$ \\
\hline 7 & 9 & & $\begin{array}{l}\text { manidipine, digoxin, esomeprazole, enelapril, januvia, duodart, } \\
\text { pravastatin, allopurinol, sintrom }\end{array}$ & $80 / 100$ & $28 / 30$ \\
\hline 8 & 8 & $\begin{array}{l}\text { lantus, adiro, furosemide, Brimica, Profer, Omeprazole, Proco- } \\
\text { ralan, Quetiapine }\end{array}$ & 9 & $95 / 100$ & $30 / 30$ \\
\hline 9 & 4 & mirtazapine, lexatin, irbesartan & 6 & $65 / 100$ & $26 / 30$ \\
\hline 10 & 13 & walker & adiro, nexium, bisoprolol, quetiapine, atrovent & $75 / 100$ & $26 / 30$ \\
\hline
\end{tabular}

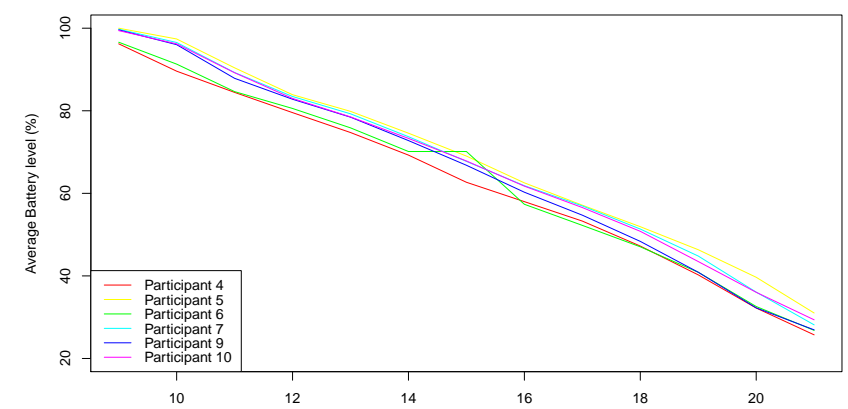

Fig. 4. Battery discharge curve for 6 participants in one day (information obtained from summary sent).

participants attended to the nursery to wear the smartband on and off everyday, and ii) as this is a prototype sometimes there are problems of communications between the smartbands and the Gateway.

\section{Conclusion and future work}

This work, presents a low-cost, robust, non-invasive and easy-to-use physical activity monitoring kit based on sensorized wristbands. The preliminary results show a good behaviour of the different hardware and software elements.

As this is is just a prototype the potential improvements are: i) The use of the kit in other kind of studies like fragility studies for example, ii) use of a more affordable gateway hardware. The Raspberry PI 3 last model can be a good option, since the model 2B gave us stability problems, iii) include a small screen for the gateway with a very simple visual interface including status and parameters of the elements in the kit, iv) Install a Data Visualization Web 


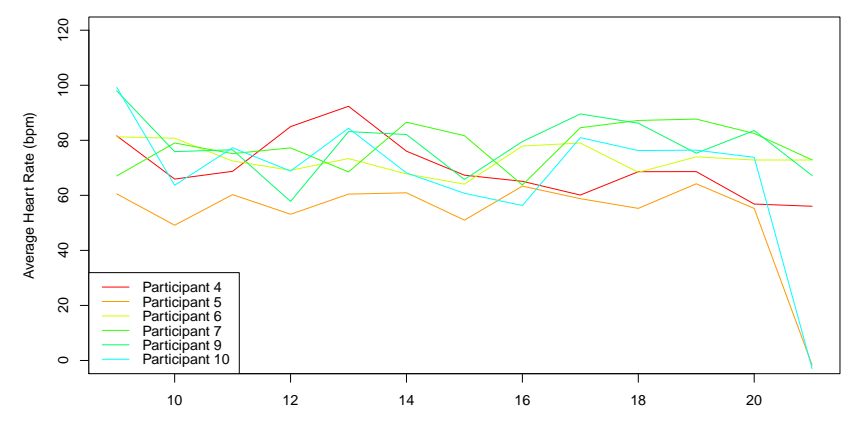

Fig. 5. Heart Rate curve for 6 participants in one day (information obtained from summary sent). At the end of the day, the HR drop is because of the WD being removed from the participants, until they are connected to the charging bases.

Application in the NUC to explode the kit locally and v) statistics rewards (activity level, evolution, ...) to encourage participants to wear the WD.

\section{Acknowledgement}

This research has been funded partially by Spanish Ministry of Economy, Industry and Competitiveness (MINECO) under grant TIN2017-84804-R and by Foundation for the Promotion of Applied Scientific Research and Technology in Asturias, under grant FC-GRUPIN-IDI2018000226.

\section{References}

1. American Geriatrics Society, B.G.S.: Ags/bgs clinical practice guideline: prevention of falls in older persons. Report, American Geriatrics Society, British Geriatrics Society (2010)

2. Bagala, F., Becker, C., Cappello, A., Chiari, L., Aminian, K., Hausdorff, J.M., Zijlstra, W., Klenk, J.: Evaluation of accelerometer-based fall detection algorithms on real-world falls. PLoS ONE 7, e37062 (2012)

3. Bianchi, F., Redmond, S.J., Narayanan, M.R., Cerutti, S., Lovell, N.H.: Barometric pressure and triaxial accelerometry-based falls event detection. IEEE Transactions on Neural Systems and Rehabilitation Engineering 18, 619-627 (2010)

4. Bourke, A.K., y G. M. Lyons, J.V.O.: Evaluation of a threshold-based triaxial accelerometer fall detection algorithm. Gait and Posture 26, 194-199 (2007)

5. Casilari, E., Santoyo-Ramón, J.A., Cano-García, J.M.: Umafall: A multisensor dataset for the research on automatic fall detection. Procedia Computer Science 110, 32-39 (2017)

6. Charlson, M., Pompei, P., Ales, K., CR., M.: Aa new method of classifying prognostic comorbidity in longitudinal studies: development and validation. J Chron Dis 40, 373-383 (1987)

7. Chaudhuri, S., y G. Demiris, H.T.: Fall detection devices and their use with older adults: A systematic review. J Geriatr Phys Ther 37(4), 178-196 (2014) 
8. Daher, M., Diab, A., Najjar, M.E.B.E., y F. Charpillet, M.A.K.: Elder tracking and fall detection system using smart tiles. IEEE Sensors Journal 17(1), 469-479 (2017)

9. De La Cal, E., Faex, M., Villar, J., Gonzalez, V.: Plataforma para el estudio de caidas y desvanecimientos en grupos de personas mayores. In: CEA BIOINGENIERA 2018 (2018)

10. Hakim, A., Huq, M.S., Shanta, S., Ibrahim, B.S.K.K.: Smartphone based data mining for fall detection: Analysis and design. Procedia Computer Science 105, 46-51 (2017)

11. Hassan, M.M., y H. Al-Dossari, H.S.A.: Internet of things framework for pervasive healthcare. 1st International Workshop on Emerging Multimedia Applications and Services for Smart Cities, EMASC 1 (2014)

12. Jalloul, N.: Wearable sensors for the monitoring of movement disorders. Biomedical Journal 41(4), 249 - 253 (2018)

13. Khelil, A., Shaikh, F.K., Sheikh, A.A., y H. Bojan, E.F.: Digiaid: A wearable health platform for automated self-tagging in emergency cases. In: de 4th International Conference on Wireless Mobile Communication and Healthcare. MOBIHEALTH20144th International Conference on Wireless Mobile Communication and Healthcare, MOBIHEALTH2014 (2014)

14. Khojasteh, S., Villar, J., de la Cal, E., Gonzlez, V., Sedano, J.: Fall detection analysis using a real fall dataset. Advances in Intelligent Systems and Computing 771, 334-343 (2019)

15. Khojasteh, S., Villar, J., Chira, C., Gonzlez, V., de la Cal, E.: Improving fall detection using an on-wrist wearable accelerometer. Sensors (Switzerland) 18(5) (2018)

16. Khojasteh, S., Villar, J., De La Cal, E., Gonzalez, V., Tan, Q., Kiadi, M.: A discussion on fall detection issues and its deployment: When cloud meets battery. In: 2018 3rd IEEE International Conference on Cloud Computing and Big Data Analysis, ICCCBDA 2018. pp. 112-115 (2018)

17. Leutheuser, H., y B. M. Eskofier, D.S.: Hierarchical, multi-sensor based classification of daily life activities: comparison with state-of-the-art algorithms using a benchmark dataset. PLoS ONE 8(10) (2013)

18. Maglogiannis, C.D., Maglogiannis, I.: Bringing iot and cloud computing towards pervasive healthcare. In: de 6th International Conference on Innovative Mobile and Internet Services in Ubiquitous Computing. IMIS 2012 (2012)

19. Rahimi, M.R., Ren, J., Liu, C.H., y N. Venkatasubramanian, A.V.V.: Mobile cloud computing: a survey, state of art and future directions. Mobile Networks and Applications 19(2), 133-143 (2014)

20. Sabatini, A.M., Ligorio, G., Mannini, A., y L. Pinna, V.G.: Prior-to- and postimpact fall detection using inertial and barometric altimeter measurements. IEEE Transactions on Neural Systems and Rehabilitation Engineering 24(7) (2016)

21. Sorvala, A., Alasaarela, E., Sorvoja, H., Myllyla, R.: A two-threshold fall detection algorithm for reducing false alarms. In: Proceedings of 2012 6th International Symposium on Medical Information and Communication Technology (ISMICT) (2012)

22. Vergara, P.M., Cal, E., Villar, J.R., González, V.M., Sedano, J.: An iot platform for epilepsy monitoring and supervising. Journal of Sensors 2017, 18 (2017)

23. Villar, J.R., Vergara, P., Menéndez, M., Cal, E., González, V.M., Sedano, J.: Generalized models for the classification of abnormal movements in daily life and its applicability to epilepsy convulsion recognition. International Journal of Neural Systems (2016), accepted for publication 